\title{
Latest results from the MEG experiment
}

\author{
M. De Gerone on behalf of the MEG Collaboration \\ Università di Roma "La Sapienza" and INFN, Sezione di Roma-Rome, Italy
}

ricevuto il 31 Agosto 2012

\begin{abstract}
Summary. - We present here the latest results on the lepton-flavour-violating (LFV) decay $\mu \rightarrow e \gamma$ based on an analysis of the data collected by the MEG detector at the Paul Scherrer Institute (PSI) in 2009 and 2010. The likelihood analysis of the combined data sample, corresponding to a total of $1.8 \times 10^{14}$ muon decays, provides a $90 \%$ CL upper limit of $2.4 \times 10^{-12}$ on the $\mu \rightarrow e \gamma$ branching ratio, constituting the most stringent limit on the existence of this decay to date.
\end{abstract}

PACS 13.35.Bv - Decays of muons.

PACS 11.30.Hv - Flavour symmetries.

\section{1. - Introduction}

In the Standard Model (SM), the $\mu^{+} \rightarrow e^{+} \gamma$ decay is forbidden by the lepton flavour conservation rule. Also including neutrinos mass and mixing, the corresponding Branching Ratio (BR) would be proportional to $\left(\frac{m_{\nu}}{M_{W}}\right)^{2}$, (where $m_{\nu}$ is the neutrino mass and $m_{W}$ the $W$ boson mass $)$, thus resulting negligible $\left(<10^{-52}\right)$. On the other hand, most theories proposed as SM extensions (e.g., SUSY) predict a much larger BR. As an example, in [1] the $\mathrm{BR}\left(\mu^{+} \rightarrow e^{+} \gamma\right)$ is predicted in the range $10^{-12}-10^{-14}$ that could be accessible by a high precision experiment, like MEG (Mu to Electron and Gamma) [2] Observation of this decay would be a definitive proof of new physics beyond the SM. The aim of MEG is to measure this BR down to a few times $10^{-13}$ improving the current limit of $1.2 \cdot 10^{-11}$ fixed by the MEGA collaboration [3].

\section{2. - The MEG experiment at PSI}

2 1. Signal and background. - The event signature of a $\mu \rightarrow e \gamma$ decay at rest is a two-bodies final state, with a positron and a photon emitted in time coincidence, moving collinearly back-to-back with their energies equal to half the muon mass $\left(m_{\mu} / 2=\right.$ $\left.52.8 \mathrm{MeV} / \mathrm{c}^{2}\right)$.

There are two major background sources [4]. The first one is the physical background coming from the radiative muon decay $\mu^{+} \rightarrow e^{+} \nu_{e} \bar{\nu}_{\mu} \gamma(\mathrm{RMD}, \mathrm{BR}(\mathrm{RMD}) \sim 1.2 \%$ of the standard Michel decay for $E_{\gamma}>10 \mathrm{MeV}$ ), the other one is an accidental coincidence 

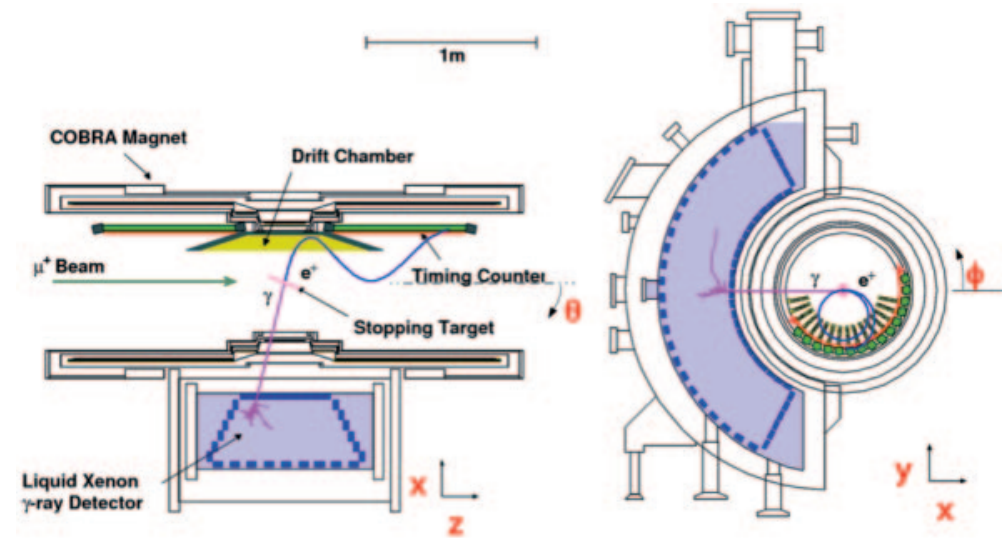

Fig. 1. - Side and front view of the MEG experiment detector layout.

of a positron from a Michel decay, $\mu^{+} \rightarrow e^{+} \nu_{e} \bar{\nu}_{\mu}$, with a high energy photon, coming from RMD, bremsstrahlung or $e^{+} / e^{-}$annihilation in flight. While the signal and RMD rates are proportional to the muon rate $R_{\mu}$, the accidental background grows as $R_{\mu}^{2}$, thus becoming the limiting factor of the experiment. Thus, usage of a continuous $\mu$ beam and implementation of a detector with cutting edge resolutions are mandatory.

2'2. Experimental apparatus. - The MEG experiment is running at the Paul Scherrer Institute (Villigen, $\mathrm{CH}$ ) where the world's most intense continuous $\mu$-beam (up to $3 \times$ $10^{8} \mu / \mathrm{s}$ ) is available. The beam is focused to the target region by a system of magnetic lenses, that has also the purpose to reduce the positron contamination. The target consists of a thin plastic foil fixed at the centre of a quasi-solenoidal superconductive magnet, called COBRA (COnstant Bending RAdius [5]). The magnetic field provided by COBRA allows the momentum selection of positrons in the 40-55 MeV energy range with cyclotron orbits smaller than $40 \mathrm{~cm}$ of diameter. Moreover, the positron bending radius is independent of the emission angle and the transversus momentum is adiabatically transferred in the longitudinal direction allowing a faster removal of positrons from the spectrometer central section. This minimize the multiple hits of those positrons that are emitted at large angles on the tracking (Drift Chambers, DC [6]) and timing (Timing Counter, TC [7]) detectors, allowing an easier track reconstruction and a better detector efficiency. While all the positrons are bounded inside the magnet volume, the emitted photons pass through the thin magnet wall and reach the liquid Xenon calorimeter (LXe), that consists of a volume of $\sim 0.9 \mathrm{~m}^{3}$ of liquid Xenon readout by photomultiplier tubes (PMTs) [8]. All the photon kinematic variables can be reconstructed using the LXe PMTs signals only. The trigger tree takes advantage of the information coming from the fast detectors (LXe and TC), with cuts based on timing, direction and energies of the reconstructed particles [9]. Signals from all detector are digitized by a $1.6 \mathrm{GHz}$ sampling chip developed at PSI, based on the Domino Ring Sampler (DRS) [10]. A sketch of the MEG experimental layout is shown in fig. 1.

\section{3. - Data analysis and results from run $2009 / 2010$}

The data analysis is based on a "blind analysis" technique in order to avoid any possible bias in results. The analysis algorithms are calibrated using a large data sample 
TABLE I. - Summary of the best estimate and confidence intervals at $90 \%$ CL for 2009, 2010 and $2009+2010$ data samples.

\begin{tabular}{|cccc|}
\hline Data sample & Best estimate & Lower limit & Upper limit \\
\hline 2009 & $3.2 \times 10^{-12}$ & $1.7 \times 10^{-13}$ & $9.6 \times 10^{-12}$ \\
2010 & $-9.9 \times 10^{-13}$ & - & $1.7 \times 10^{-12}$ \\
$2009+2010$ & $-1.5 \times 10^{-13}$ & - & $2.4 \times 10^{-12}$ \\
\hline
\end{tabular}

in the side-bands outside the blinding box. Moreover, also the background level in the signal region can be estimated by the analysis of the side-band regions, being the main source of background the accidental one. The number of signal, RMD and accidental events in the signal region is extracted by means of an extended maximum-likelihood fit to the five observables defining the event. The fit is performed in the analysis region, defined as $48 \mathrm{MeV}<E_{\gamma}<58 \mathrm{MeV}, 50 \mathrm{MeV}<E_{e}<56 \mathrm{MeV},\left|t_{e \gamma}\right|<0.7 \mathrm{~ns},\left|\theta_{e \gamma}\right|<50 \mathrm{mrad}$ and $\left|\phi_{e \gamma}\right|<50 \mathrm{mrad}$. The likelihood function is defined as

$$
\begin{aligned}
& \mathcal{L}\left(N_{\mathrm{SIG}}, N_{\mathrm{RMD}}, N_{\mathrm{BG}}\right)= \\
& \frac{e^{-N}}{N_{\text {obs }} !} e^{-\frac{\left(N_{\mathrm{RMD}}-\left\langle N_{\mathrm{RMD}}\right\rangle\right)^{2}}{2 \sigma_{\mathrm{RMD}}^{2}}} e^{-\frac{\left(N_{\mathrm{BG}}-\left\langle N_{\mathrm{BG}}\right\rangle\right)^{2}}{2 \sigma_{\mathrm{BG}}^{2}}} \prod_{i=1}^{N_{\mathrm{obs}}}\left(N_{\mathrm{SIG}} S\left(\vec{x}_{i}\right)+N_{\mathrm{RMD}} R\left(\vec{x}_{i}\right)+N_{\mathrm{BG}} B\left(\vec{x}_{i}\right)\right),
\end{aligned}
$$

where $N_{\mathrm{SIG}}, N_{\mathrm{RMD}}$, and $N_{\mathrm{BG}}$ are the number of signal, RMD and accidental events respectively, $S, R$ and $B$ are their respective probability density functions (PDF) and $\vec{x}_{i}=\left\{E_{\gamma}, E_{e}, t_{e \gamma}, \theta_{e \gamma}, \phi_{e \gamma}\right\}$ is the vector of observables for the $i$-th event. $N_{\text {obs }}$ is the total number of events observed in the analysis window and $N=N_{\mathrm{SIG}}+N_{\mathrm{RMD}}+N_{\mathrm{BG}}$. The PDFs for signal, RMD and accidental background are determined as follow:

- $S$ is given by the product of the statistically independent PDFs for the five observables, each defined by the their corresponding detector resolutions, measured on dedicated calibration runs;

- $R$ is the product of the PDF for $t_{e \gamma}$, which is the same as that for the signal, and the PDF for the other correlated variables, obtained by folding the theoretical spectrum with the detector resolutions;

- $B$ is determined by the product of the background spectra for each variable, measured on the side-bands.

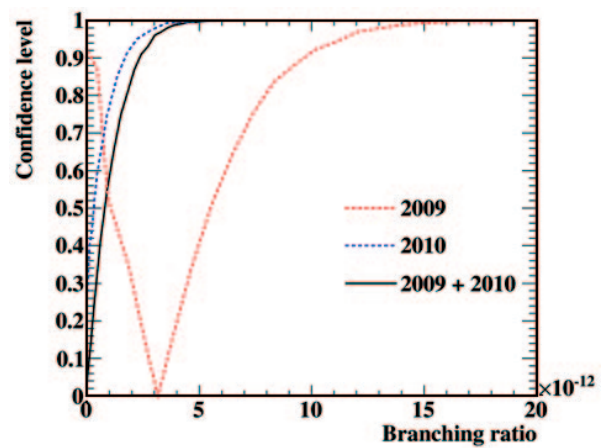

Fig. 2. - Confidence level curves for 2009, 2010 and 2009+2010 data samples. 
A frequentistic approach with a profile likelihood-ratio ordering [11] is used to compute the confidence intervals on $N_{\mathrm{SIG}}$. The upper limit on the BR is then obtained by normalizing the upper limit on the number of signals to the total number of Michel decays, counted simultaneously with the signal, using the same analysis cuts. Such a normalization scheme has the great advantage to be independent from the instantaneous rate of the beam and nearly insensitive to the positron acceptance and efficiencies of DC. The sensitivity of the measure, defined as the 90\% CL upper limit of the BR averaged over a set of toy $\mathrm{MC}$ experiment with a background only hypothesis, is calculated to be $3.3 \times 10^{-12}, 2.2 \times 10^{-12}$ and $1.6 \times 10^{-12}$ for the 2009,2010 and $2009+2010$ data set, respectively. These numbers are consistent with the BR upper limit observed at the side-bands.

Finally, the combined $2009+2010$ data sample analysis gives the most stringent BR upper limit on $\mu \rightarrow e \gamma$ of $2.4 \times 10^{-12}$ [2], improving the previous upper limit by a factor 5. The $90 \%$ CL intervals as well as the best estimate of the branching ratio for 2009 and 2010 data separately are given in table I, while the confidence level curves are shown in fig. 2. A lower limit was set by the analysis of the 2009 data sample, but its statistical significance is low, being still consistent with the background-only hypothesis with a probability of $8 \%$. The systematic uncertainties for the PDF parameters and the normalization factor are taken into account in the calculation of the confidence intervals by fluctuating the PDFs accordingly to their uncertainties.

\section{4. - Conclusion and perspectives}

The MEG experiment looks for the LFV decay $\mu \rightarrow e \gamma$, with an unprecedented sensitivity. The analysis of the data taken by the MEG detector during the 2009 and 2010 runs gives as result $\mathrm{BR}(\mu \rightarrow e \gamma)<2.4 \times 10^{-12}$ thus fixing the most stringent limit on this BR to date. The 2011 run is completed, while 2012 one is ready to start. We plan to reach the sensitivity region of $O\left(10^{-13}\right)$ with the analysis of the full data sample. Moreover, an R\&D work on detector upgrade has already started, aiming to improve the search sensitivity up to few $10^{-14}$.

\section{REFERENCES}

[1] BArbieri R. et al., Nucl. Phys. B, 445 (1995) 219.

[2] Adam J. et al. (MEG Collaboration), Phys. Rev. Lett., 107 (2011) 171801.

[3] Brooks M. L. et al. (MEGA Collaboration), Phys. Rev. Lett., 83 (1999) 1521.

[4] Kuno Y. and Okada Y., Rev. Mod. Phys., 73 (2001) 151.

[5] Ootani W. et al., IEEE Trans. Appl. Supercond., 14 (2004) 568.

[6] Hildebrandt M. et al., Nucl. Instrum. Methods A, 623 (2010) 111.

[7] De Gerone M. et al., Nucl. Instrum. Methods A, 638 (2010) 41.

[8] Mihara S. et al., J. Phys.: Conf Ser., 308 (2009) 012009.

[9] Galli L., A real time glance at the lepton flavour violating decay muon to positron and gamma in the MEG experiment, $\mathrm{PhD}$ Thesis (2010).

[10] RitT S. et al., Nucl. Instrum. Methods A, 623 (2010) 486.

[11] Feldmann G. J. and Cousins R. D., Phys. Rev. D, 57 (1998) 3783. 